\title{
Effect of Write Head Movement On Magnetic Spin Domain Reversal of Nanocube Co/Pd Alloy Material Using Micromagnetic Simulation
}

\author{
Ilham Heru Baskoro ${ }^{1, a}$ and Merinda Lestari ${ }^{1}$ \\ ${ }^{1}$ Department of Physics, Faculty of Mathematics and Natural Sciences, University of Jember, \\ Jalan Kalimantan No. 37, Jember 68121, Indonesia \\ aherubaskorowinchester@gmail.com
}

\begin{abstract}
An analysis of the effect of the write head movement on the reversal time of the domain spin with magnetic $\mathrm{Co} / \mathrm{Pd}$ on the magnetic recording layer has been carried out through micromagnetic simulation. The magnetic recording layer is modeled in the form of cubes (nanocubes) which consists of 5 domain spin. The write head, which is a transduser, moves along the domain spin to write data in the form of magnetic spins, which represent the bits on the magnetic recorder perpendicular. The results of this simulation are a profile of changes in the total magnetic field and reversal time of the domain spin when writing magnetic data for 6 nanoseconds. The calculation used in this study is an analytical calculation regarding the reversal time of the magnetic domain spin of the $\mathrm{Co} / \mathrm{Pd}$ alloy material. The formulation for calculating the reversal time of domain-spin magnetization is a combination of graphical analysis and analytical calculations with visualization of the magnetic spin configuration that consisting of 5 domains spin. This simulation was carried out using the finite element method and obtained a saturation field value of the magnetic alloy $\mathrm{Co} / \mathrm{Pd}\left(\mathrm{H}_{\mathrm{s}}\right)$ material of $2.5 \times 10^{5} \mathrm{~A} / \mathrm{m}$ and a write head $(\mathrm{Hwh})$ field that must be applied to the magnetic recording layer in order to reverse the uniform domain spin is $7.3 \times 10^{6}$ $\mathrm{A} / \mathrm{m}$. Each size of the domain spin requires a different write head, the smaller the nanocube size, the greater the write head field applied to the magnetic recording layer. Meanwhile, the effective write head field amplitude that is suitable for the $20 \mathrm{~nm}$ domain spin is $8.3 \times 10^{6} \mathrm{~A} / \mathrm{m}$. A significant change in the total field occurs when the domain spin reverses 3 times in the first domain spin $\left(n_{1}\right)$, the third domain spin $\left(n_{3}\right)$ and the fifth domain spin $\left(n_{5}\right)$. The total field value when $t=0.42$ ns ( first domain spin reversal) is $73.69376 \mathrm{~A} / \mathrm{m}$, then the total field at $\mathrm{t}=0.42 \mathrm{~ns}$ (third domain spin reversal) is $3443.197 \mathrm{~A} / \mathrm{m}$ and the current total field $t=0.42 \mathrm{~ns}$ (fifth domain spin reversal) of $5480.696 \mathrm{~A} / \mathrm{m}$.
\end{abstract}

Keywords: Write Head, Perpendicular Magnetic Recorder, Finite Element Method, Domain Spin, Saturation Field $\left(\mathrm{H}_{\mathrm{s}}\right)$.

\section{Introduction}

One of the potential materials for spintronic device applications is ferromagnetic materials. Naturally, ferromagnetic materials have spontaneous magnetizing property known as a magnetic domain. The configuration of the spin structure in the magnetic domain can be controlled using a magnetic field. as a result, there is a change in the configuration of the spin direction in the magnetic domain which is interpreted as a process of changing the magnetization (magnetization reversal). The changes in magnetization in ferromagnetic materials are fundamental to magnet based data storage devices [1]. Ferromagnetic materials have a domain wall which is formed from the competition between exchange energy and anisotropy enenrgy. Exchange energy is used to thicken the domain wall. However, the thicker domain wall will cause the spin direction transisition. This will increase the anisotropy effect. If this effect is increased continously, it will make the domain wall thinner. 
The competition between exchange energy and anisotropy energy is characterized by the exchange length.

$I_{e x}=\sqrt{\frac{2 A}{\mu_{0} M_{S}^{2}}}$

Where, $I_{e x}$ is exchange length $(m)$, A is exchange stiffness constant $(J / m), \mu_{0}$ is the air permeability $\left(J / A^{2} m\right), M_{S}$ is saturation magnetization $(A / m)$ [2].

On the hard disk, information is generally stored on a ferromagnetic granular film that is in a magnetized state. The granular medium is the composition of the seeds, which are located with the same structure crystal that making the magnetization parallel straight to one axis, which is called the easy axis. A systematic understanding of the magnetization dynamics in write heads is essential in understanding the effects of perpendicular magnetic recording (PMR) to increase bit density and data rates. However, recently it is only possible to compute the magnetodynamics of the overall system interaction of the write dead, domain spin data media (DL), and soft undermedia (SUL) [3].

The biggestt problem at this time is that perpendicular magnetic recording is limited by trilemma caused by superparamagnetic limits, namely thermal stability, grains size, and writing capability when data storage density is increased. The smaller size of the grains, a larger write current consumption is required. In the cross-secion configuration of the bit that perpendicular requires HAMR (Heat Assisted Magnetic Recording) technology, which uses illumination or light from a laser to write data on the medium. HAMR is an attempt to take advantage of the dependency of high temperature from magnetic anisotropy, by increasing the temperature can reduce the coercivity of the medium significantly so that data recording can appear with a small write field [4].

Another research that has been done is the micromagnetic study of patterned media. In this study, a material for perpendicular recording medium was used, namely the multimedia structure of $\mathrm{Co} / \mathrm{Pd}$ which has the perpendicular orientation to the same magnetic media with Co medium with thickness $(0.2-0.3 \mathrm{~nm})$. The magnetic recording medium for patterned media is based on the multimedia Co/Pd island with a domain spin microstucture measuring $10 \mathrm{~nm}$ and thick $15 \mathrm{~nm}$. The size of the separated magnetic island is about $30 \times 30 \mathrm{~nm}^{2}$ and the bits separated by a $60 \mathrm{~nm}$ longitudinal peak and a $250 \mathrm{~nm}$ radial peak yield a storage density of 180 Gbit/in ${ }^{2}[5]$.

\section{Materials and Methods}

The initial stage in this research is that the domain spin configuration is modeled in $3 \mathrm{D}$ and the grains geometry is determined according to the separation gap between each nanoisland, which is $10 \mathrm{~nm}$. In this study, the nanoisland size was $20 \mathrm{~nm}$ then varied $(5-20 \mathrm{~nm})$. 


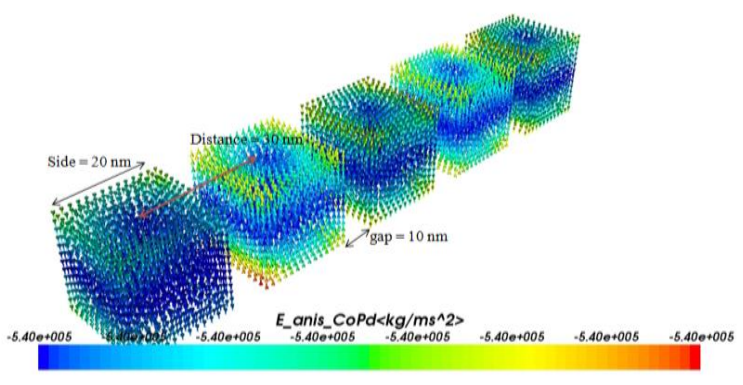

Figure 1. The size of perpendicular domain spin (nanometer)

The research was conducted using publicly licensed software, namely NMAG (Nano Magnetic) which can be run on the GNU/ Linux operating system. The method used in this research is the finite element method. The basic concept of the finite element method is to solve a problem by dividing the object of analysis into finite small parts. These small pieces are then analyzed and the results are recombined to find a solution for the whole area. This micromagnetic simulation uses the formulation of time differential equations order-one, as follows:

$\frac{\partial \boldsymbol{M}}{\partial t}=-\gamma_{L} \boldsymbol{M} \times \boldsymbol{H}_{\text {eff }}-\frac{\alpha_{L}}{M_{S}} \boldsymbol{M} \times\left(\boldsymbol{M} \times \boldsymbol{H}_{\text {eff }}\right)$

The variation of the write head field is $1.0 \times 10^{6} \mathrm{~A} / \mathrm{m}, 7.2 \times 10^{6} \mathrm{~A} / \mathrm{m}, 7.3 \times 10^{6} \mathrm{~A} / \mathrm{m}, 8.3 \times 10^{6} \mathrm{~A} / \mathrm{m}$ to $83 \times 10^{6} \mathrm{~A} / \mathrm{m}$. While the geometry of the material used is $20 \mathrm{~nm}$ for the nanocube side.

Nanocube-shaped Co/Pd material parameters can be seen in Table 1.

Table 1. Nanocube-shaped Co/Pd material parameters

\begin{tabular}{cc}
\hline Parameter & Value \\
\hline$M_{s}$ & $4.2126 \times 10^{5} \mathrm{~A} / \mathrm{m}$ \\
$A$ & $30 \times 10^{-72} \mathrm{~J} / \mathrm{m}$ \\
$K$ & $5.4 \times 10^{5} \mathrm{~J} / \mathrm{m}^{3}$ \\
$l_{e x}$ & $16.4 \mathrm{~nm}$ \\
\hline
\end{tabular}

After inputting all the $\mathrm{Co} / \mathrm{Pd}$ material parameters, the output data will be obtained in the form of a .vtk file and a .ndt file. The .vtk file then used to visualize the domain structure. The visualization can show the direction of spin and the magnetization value of the Co/Pd material. Furthermore, the results are represented in the form of a graph of the total magnetic field relationship to time $\left(\mathrm{H}_{\text {tot }} \mathrm{t}\right)$ from the .ndt file. In addition, a domain spin reversal time analysis was carried out related to the dynamics of the write head movement due to the influence of the amplitude variation of the write head field, on the simple moving of the write head phenomenon by varying the write head field on the same domain spin geometry and finding the best write head field for each size.

\section{Results and Discussion}

\section{Analysis of the relationship between the saturation field and the write head field to be applied}

The relation between the two was analyzed based on two different simulations, namely the simulation of the hysteresis curve of the magnetic $\mathrm{Co} / \mathrm{Pd}$ material and the simulation of the effect of write head movement on the magnetic recording medium. In this discussion, an 
explanation of the saturation field of magnetic $\mathrm{Co} / \mathrm{Pd}$ material on the hysteresis curve is presented and its relation to the change in the total field (noise) in the magnetic recording medium during the data writing process for 6 nanoseconds.

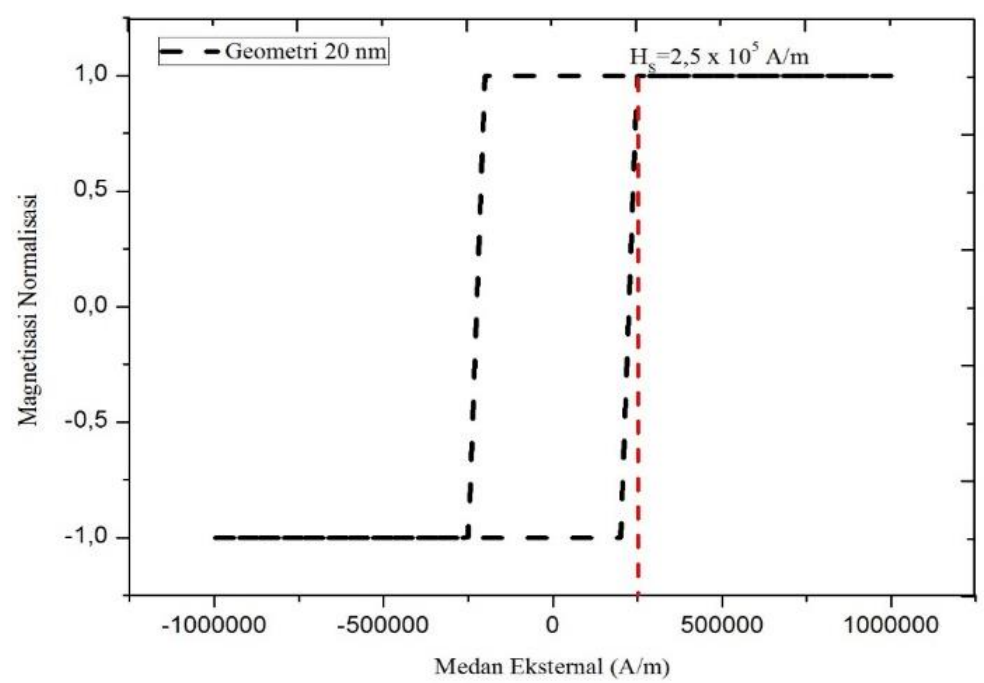

Figure 2. Hysteresis curve of the domain spin of $\mathrm{Co} / \mathrm{Pd}$ magnetic material with side size of 20 $\mathrm{nm}$ nanocube.

Figure 2 is a hysteresis curve showing the $\mathrm{H}_{\mathrm{s}}$ saturation field of the Co/Pd magnetic material as many as $2.5 \times 10^{5} \mathrm{~A} / \mathrm{m}$. The saturation field $\mathrm{H}_{\mathrm{s}}$ is the amount of field needed to achieve saturation (saturation state 0 and as initial information in analyzing how much the write head field needs to be applied to $20 \mathrm{~nm}$ Co/Pd grains to cause a magnetization reversal domain spin. The applied write head field or the $\mathrm{H}_{\text {trand }}$ write head field must satisfy $\mathrm{H}_{\text {trand }}>\mathrm{H}_{\mathrm{s}}$ so that the write head field is not applied below the saturation field value. The simulation of the effect of write head movement on the magnetic recording medium is carried out by varying the amplitude of the write head field to obtain information abaout the domain spin response by applying a write head field to the magnetic recording medium of $2.5 \times 10^{5}-7.2 \times 10^{6}(\mathrm{~A} / \mathrm{m})$. Figure 3 shows a reversal of the domain spin magnetization which is irregular and the noise in the medium that tends to be stable. The applied write head field is then enlarged to $7.3 \times 10^{6} \mathrm{~A} / \mathrm{m}$, so that the regular domain spin magnetization reversal occurs as shown in Figure 4.6. this is done to obtain the effective write head field and domain spin reversal time. Figures 3-5 show the reversal orientation on the domain spin with spin down.

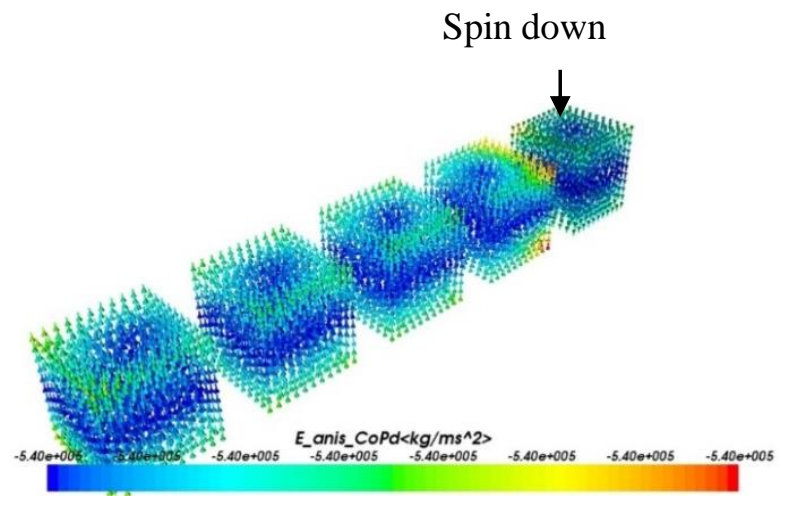


Figure 3. First spin (island) domain reversal at time ( $\mathrm{t}=0.42 \mathrm{~ns}$ ) on the magnetic recording medium

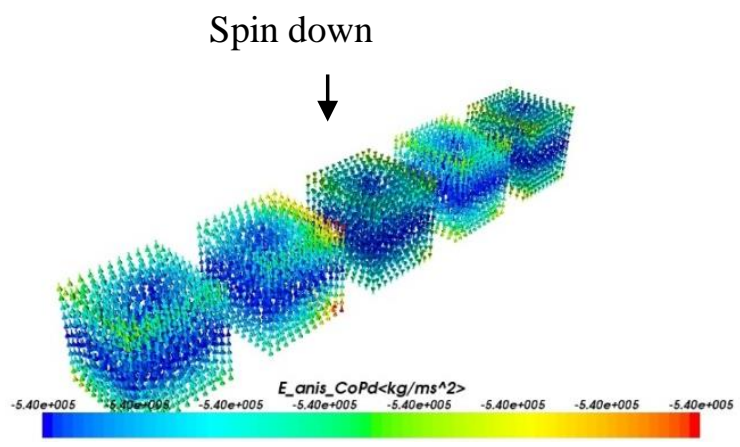

Figure 4. Second spin (island) domain reversal at time ( $\mathrm{t}=2.42 \mathrm{~ns}$ ) on the magnetic recording medium

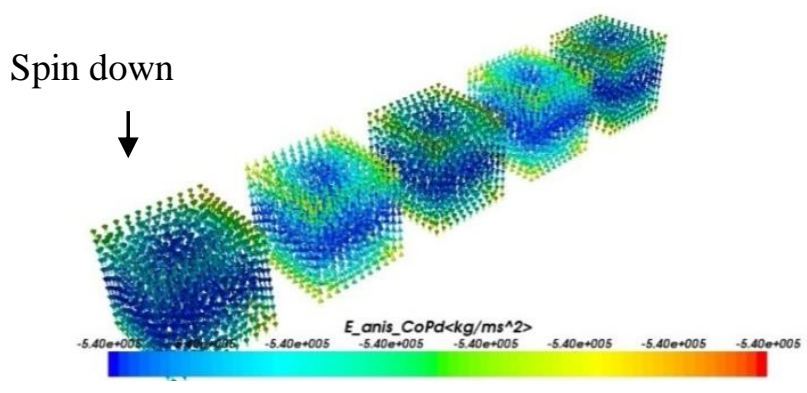

Figure 5. Third spin (island) domain reversal at time ( $\mathrm{t}=4.42 \mathrm{~ns})$ on the magnetic recording medium

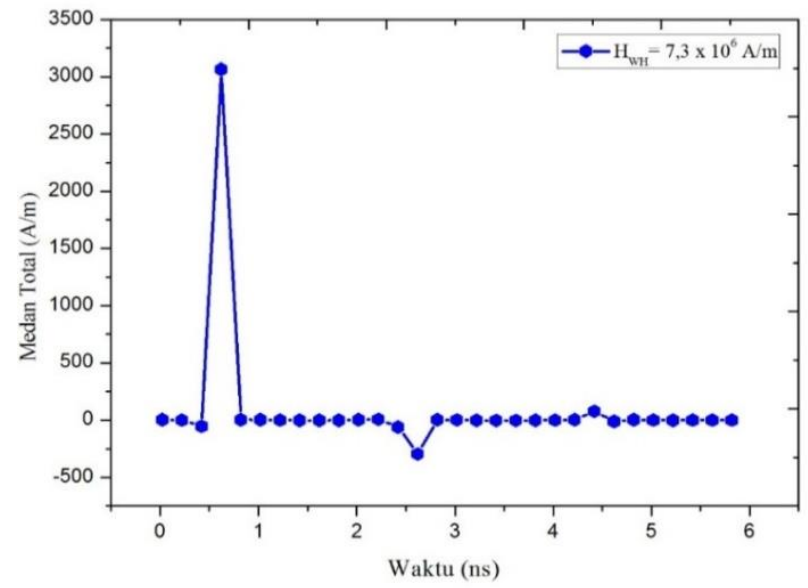

Figure 6. Graph of the total field of domain spin change on recording media for 6 nanoseconds with a write head field amplitude of $7.3 \times 10^{6} \mathrm{~A} / \mathrm{m}$ 


\section{Application of the best Write Head Field for Each Side Size Variation of Nanocube Co/Pd}

This section discusses the application of the best write head fields for each size variation of nanocubes or grains. The small nanocube size is needed in perpendicular magnetic recording technology which requires a larger write head field consumption because the coercivity of the perpendicular recording medium is greater than that of the magnetic recording medium in general. Below is the variation in the size of the Co/Pd nano cube under the critical geometry of the magnetic Co/Pd material.

a) Application of the best write head field for the $5 \mathrm{~nm}$ side sized nanocube

Spin down

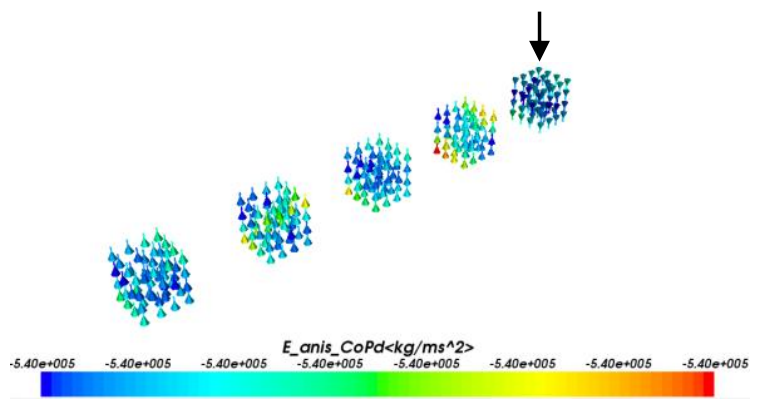

Figure 7. The first domain spin reversal of the magnetic recording medium at $t=0.42 \mathrm{~ns}$ Spin down

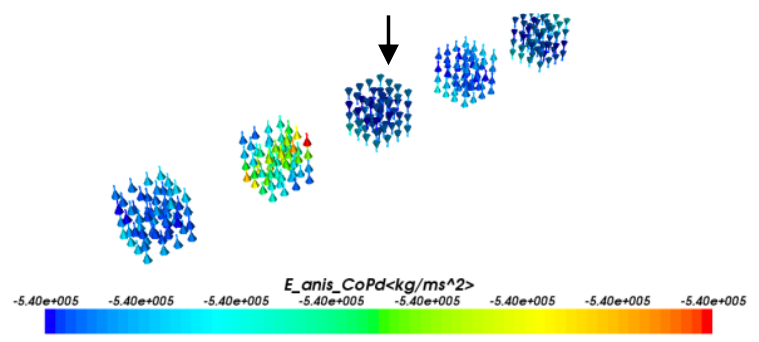

Figure 8. The second domain spin reversal of the magnetic recording medium at $t=2.42 \mathrm{~ns}$

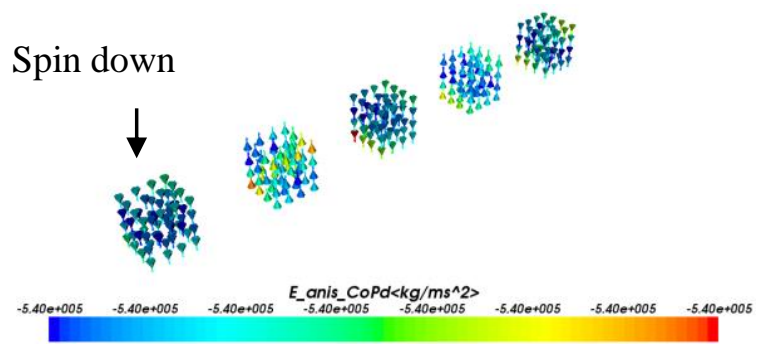

Figure 9. The third domain spin reversal of the magnetic recording medium at $\mathrm{t}=4.42 \mathrm{~ns}$ 


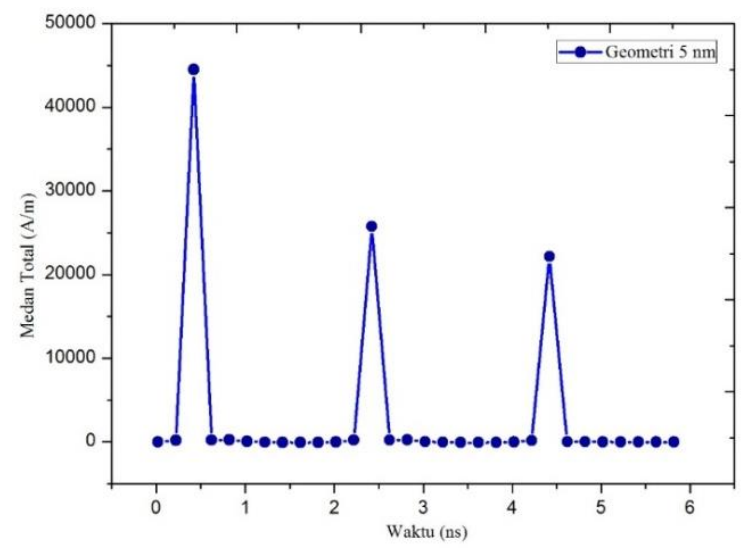

Figure 10. Graph of the total field change againts the time for 6 nanoseconds on a $5 \mathrm{~nm}$ domain spin with a write head field of $9.5 \times 10^{7} \mathrm{~A} / \mathrm{m}$

b) Application of the best write head field for the $20 \mathrm{~nm}$ side sized nanocube

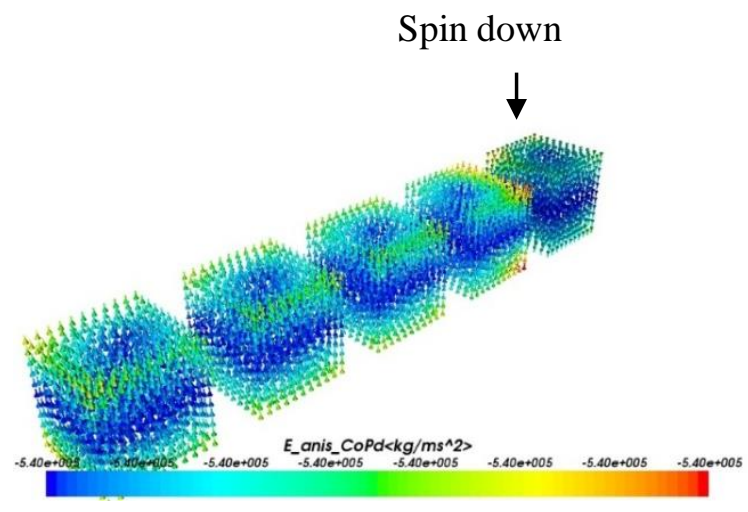

Figure 11. The first domain spin reversal of the magnetic recording medium at $t=0.42 \mathrm{~ns}$

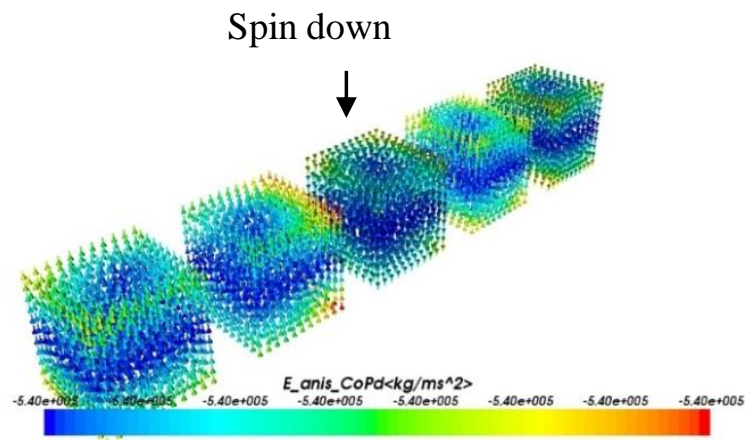

Figure 12. The second domain spin reversal of the magnetic recording medium at $t=2.42$ ns 


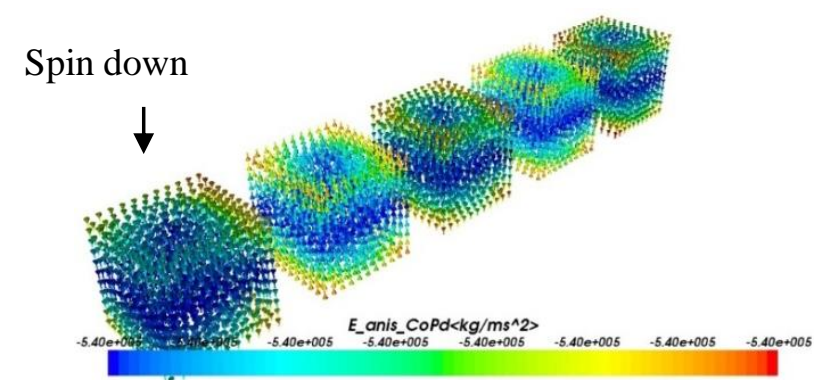

Figure 13. The third domain spin reversal of the magnetic recording medium at $t=4.42 \mathrm{~ns}$

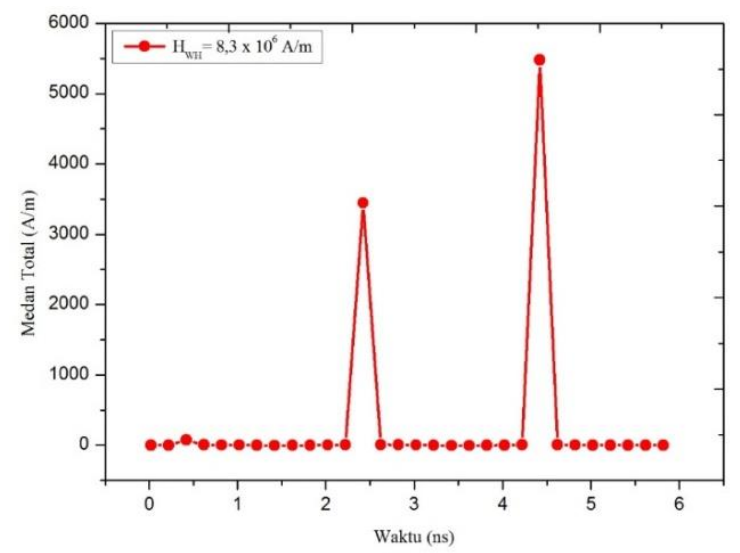

Figure 14. Graph of the total domain spin field change in recording media for 6 nanoseconds with a domain spin side of $20 \mathrm{~nm}$

Table 2. The suitability of the write head field applied to each nanocube size variation

\begin{tabular}{ccc}
\hline No. & The size of Nanocube $[\mathrm{nm}]$ & $\begin{array}{c}\text { Write head field } \\
\text { amplitude }[\mathrm{A} / \mathrm{m}]\end{array}$ \\
\hline 1 & 5.0 & $95 \times 10^{6}$ \\
2 & 7.5 & $53 \times 10^{6}$ \\
3 & 10.0 & $26 \times 10^{6}$ \\
4 & 12.5 & $17 \times 10^{6}$ \\
5 & 15.0 & $9.7 \times 10^{6}$ \\
6 & 17.5 & $8.7 \times 10^{6}$ \\
7 & 20.0 & $8.3 \times 10^{6}$ \\
\hline
\end{tabular}

In Table 2 above, it is explained that each nanocube size has the best write head field based on a graphical analysis of the system total magnetic field change and the timing diagram in accordance with the previous discussion. Based on the above discussion, it has been researched and obtained the best write head field for each grains size of the Co/Pd allor material, where the graph of the total magnetic field changes only changes significantly when the domain spin reversal occurs and during the transition time, the total magnetic field value is relatively small hence negligible, or relatively low noise from the magnetic recording medium. Basically, the substance of the main study regarding noise in magnetic recording medium is related to the granularity of the medium. The total field value of the magnetic 
recording medium which changes due to a given or applied write head field can be specifically described, because the recording medium is a thin medium or thin film that has strong magnetic interactions and tightly arranged, an effective write head field is needed so that the total magnetic field changes significantly when the domain spin reversal occurs. The size of the grains geometry of the Co/Pd alloy material required the smallest size, in this study the nanocube geometry (5-20) nm was examined.

Table 3. The average domain spin reversal times for each domain spin size

\begin{tabular}{ccccc}
\hline \multirow{2}{*}{ No. } & The size of spin & \multicolumn{3}{c}{ Average domain spin reversal time [s] } \\
\cline { 3 - 5 } & domain [nm] & Pertama & Kedua & Ketiga \\
\hline 1 & 5.0 & 0.42 & 2.42 & 4.42 \\
2 & 7.5 & 0.42 & 2.42 & 4.42 \\
3 & 10.0 & 0.42 & 2.42 & 4.42 \\
4 & 12.5 & 0.42 & 2.42 & 4.42 \\
5 & 15.0 & 0.62 & 2.62 & 4.62 \\
6 & 17.5 & 0.42 & 2.42 & 4.42 \\
7 & 20.0 & 0.42 & 2.42 & 4.42 \\
\hline
\end{tabular}

\section{Conclusions}

The simulation results produce that the write head field appied to the magnetic recording medium must be greater than the saturation field of the Co/Pd magnetic material where $\mathrm{H}_{\mathrm{s}}=2.5$ $\times 10^{5} \mathrm{~A} / \mathrm{m}$ and to achieve a regular domain spin reversal, the write head of $\mathrm{H}_{\mathrm{WH}}=7.3 \times 10^{6} \mathrm{~A} / \mathrm{m}$ (about 30 times as big) is needed. The greater the amplitude value of the write head field will affect the change in the total magnetic field and the time of domain spin reversal. The write head field amplitude is given starting from $1.0 \times 10^{6} \mathrm{~A} / \mathrm{m}$ until it reaches an effective write head field of $\mathrm{H}_{\mathrm{WH}}=8.3 \times 10^{6} \mathrm{~A} / \mathrm{m}$. The smaller the size of the Co/Pd grains (nanocubes), the greater the write head field that needs to be applied to the magnetic recording layer to cause a magnetization reversal in a regular domain spin.

\section{References}

[1] A T Widodo, 2013, Micromagnetic Study of Domain Structure Dynamics in Ferromagnetic Materials Py, Ni, Fe, and Co Nanosprehe Model, Thesis, Universitas Indonesia Press, Depok, Indonesia.

[2] S Chikazumi and S H Charap, 1964, Physics of Magnetism, John Wiley \& Sons, Inc.

[3] L Schrefl, M E Schabes, D Suess and M Stehno, 2004, Dynamic micromagnetic write head fields during magnetic recording in granular media, IEEE Trans. Magn., vol. 40, page 23412343.

[4] T Rausch, 2002, Optical Data Storage, Conf. SPIE Proc. 4342, 502

[5] J Fidler, K H Oh, T Schrefl, D Suess, and J Lee, 2007, Magnetic characteristics of ferromagnetic nanotube, Journal of Magnetism and Magnetic Materials, 310, page 24452447. 